\title{
LOCAL ERGODICITY OF NONPOSITIVE CONTRACTIONS ON $C(X)$
}

\author{
ROBERT E. ATALLA
}

\begin{abstract}
Let $T$ be an operator on $C(X), X$ compact, with $\|T\| \leqslant 1$, and suppose $T$ has a nowhere vanishing invariant function $\psi^{-1}$. The operator $R$ defined by $R f=$ $T\left(f \psi^{-1}\right) \psi$ is (a) "locally" a Markov operator, and (b) (locally) strongly ergodic iff $T$ is. This is used to prove Sine's local strong ergodicity theorem without assuming that $T$ is positive.
\end{abstract}

1. Introduction. $C(X)$ will stand for the scalar valued continuous functions on $X$, where the scalar field can be taken as either the real or complex numbers, and $T$ will be a linear operator with $\|T\| \leqslant 1$. Let $T^{*}$ be the adjoint of $T$ and write

$$
F(T)=\{f \in C(X): T f=f\} \text { and } F\left(T^{*}\right)=\left\{m \in C(X)^{*}: T^{*} m=m\right\} .
$$

$T$ is called strongly ergodic if there exists a projection $P$ such that $T_{n} f \rightarrow P f$ $(f \in C(X))$, where $T_{n}=(1 / n)\left(I+\cdots+T^{n-1}\right)$.

Let $M=$ closure $\cup\left\{\operatorname{supp}(|m|): m \in F\left(T^{*}\right)\right\}$. Since, as we shall prove, $f \mid M=0$ implies $T f \mid M=0, T$ induces in a natural way an operator $T_{0}$ on $C(M)\left[\mathbf{A}, \mathbf{S}_{3}\right]$. Namely, if $f \in C(M)$, let $\bar{f} \in C(X)$ be any extension of $f$, and define $T_{0} f=T \bar{f} \mid M$. $T$ is called locally strongly ergodic if $T_{0}$ is strongly ergodic on $C(M)$.

We shall later refer to Sine's criterion for ergodicity of a linear contraction $R$ on a Banach space $B\left[\mathbf{S}_{2}\right]: R$ is strongly ergodic iff $F(R)$ separates $F\left(R^{*}\right)$, i.e., if $p$ and $q$ are distinct elements of $F\left(R^{*}\right)$, then there exists $f$ in $F(R)$ such that $p(f) \neq q(f)$. More generally this result holds if $R$ satisfies

$$
\left\|R_{n}\right\| \leqslant K \quad(n=1,2, \ldots) \text { and }(1 / n)\left\|R^{n}\right\| \rightarrow 0 .
$$

(See $[\mathbf{L l}]$ and, for a simplified proof, [Sa].)

We find it convenient to state the separation criterion in a somewhat different form. Since $F\left(R^{*}\right)$ is a linear space, the criterion is equivalent to saying that if $p$ is a nonzero element of $F\left(R^{*}\right)$, then there exists $f$ in $F(R)$ with $p(f) \neq 0$. Hence $R$ is strongly ergodic iff the only element of $F\left(R^{*}\right)$ which annihilates $F(R)$ is 0 , i.e., iff $F\left(R^{*}\right) \cap F(R)^{\perp}=(0)$, where $F(R)^{\perp}=\left\{m\right.$ in $B^{*}: m(f)=0$ for all $f$ in $\left.F(R)\right\}$.

2. Preliminaries. The Radon-Nikodym derivative defined in 2.1 below was introduced in [F-R] for the special case where $T^{2}=T$. Lemma 2.2 is a generalization of Lemma 1.2 in $\S 1$ of [F-R].

Received by the editors January 1, 1981 and, in revised form, September 28, 1981, March 19, 1982, July 27, 1982 and November 5, 1982.

1980 Mathematics Subject Classification. Primary 47A35; Secondary 47B55.

Key words and phrases. Markov operator, contraction, strongly ergodic, invariant measure. 
2.1. Definition. Let $F_{1}\left(T^{*}\right)$ be the closed unit ball of $F\left(T^{*}\right)$, and let ext $F_{1}\left(T^{*}\right)$ be the set of extreme points. If $m \in \operatorname{ext} F_{1}\left(T^{*}\right)$, let $\varphi_{m}=d m / d|m|$, so $d m=\varphi_{m} d|m|$, with $\|m\|=\||m|\|=1$. (Note that $\left|\varphi_{m}\right|=1|m|$-a.e. and $d|m|=\bar{\varphi}_{m} d m$.)

2.2. Lemma. Suppose $f$ is in $F(T)$ and $m$ is in ext $F_{1}\left(T^{*}\right)$. Then $f=\left(\int f d m\right) \bar{\varphi}_{m}$ $|m|$-a.e.

Proof. First we show that $f \varphi$ is constant $|m|$-a.e. (where $\varphi=\varphi_{m}$ ). If not, then for some real $c$, the sets $A=\{x: \operatorname{Re}(f(x) \varphi(x)) \geqslant c\}$ and $B=X \backslash A$ are not $|m|$-null, say $t=|m|(A)$ and $1-t=|m|(B)$, where $0<t<1$. Then

$$
m=t\left(\left.m\right|_{A} / t\right)+(1-t)\left(\left.m\right|_{B} /(1-t)\right)=t m_{1}+(1-t) m_{2},
$$

and, for $n \geqslant 1$,

$$
m=T_{n}^{*} m=t T_{n}^{*} m_{1}+(1-t) T_{n}^{*} m_{2}
$$

By weak-* compactness of the unit ball in $C(X)^{*}$, there exists $p_{1}$ and $p_{2}$ and nets $\left\{T_{n(a)}^{*} m_{1}\right\},\left\{T_{n(a)}^{*} m_{2}\right\}$ with $T_{n(a)}^{*} m_{1} \rightarrow p_{1}$ and $T_{n(a)}^{*} m_{2} \rightarrow p_{2}$. From (*) we get

$$
m=t p_{1}+(1-t) p_{2} \quad\left(p_{1}, p_{2} \in F_{1}\left(T^{*}\right)\right) .
$$

We now show that $p_{1} \neq p_{2}$, contrary to extremeness of $m$. Clearly $T_{n(a)} f=f$ on $\operatorname{supp}(|m|)$ and, hence,

$$
\begin{aligned}
p_{1}(f) & =\lim T_{n(a)}^{*}\left(\left.m\right|_{A} / t\right)(f)=\left.\lim m\right|_{A}\left(T_{n(a)} f\right) / t \\
& =\left.\lim m\right|_{A}(f) / t=\frac{1}{t} \int_{A} f d m=\frac{1}{t} \int_{A} f \varphi d|m| .
\end{aligned}
$$

Since $\operatorname{Re}(f \varphi) \geqslant c$ on $A$ and $\left.|m|\right|_{A} / t$ is a probability measure, we have $\operatorname{Re}\left(p_{1}(f)\right) \geqslant$ $c$. Likewise $\operatorname{Re}\left(p_{2}(f)\right)<c$ and, hence, $p_{1} \neq p_{2}$.

Thus for some $k, f=k \bar{\varphi}|m|$-a.e. But $k=\int k d|m|=\int f \varphi d|m|=\int f d m$.

2.3. REMARK. It follows that if there exists $f$ in $C(X)$ as in 2.2 and $\int f d m \neq 0$, then we may assume $\varphi_{m}$ is continuous on $\operatorname{supp}(|m|)$ and vanishes off this set.

2.4. EXAmple. Lemma 2.2 can fail if $T$ is not a contraction. For example, let $X=\{1,2\}$, so $C(X)$ is (algebraically) the same as either $R^{2}$ or $C^{2}$. We define $T$ by the matrix $\left[\begin{array}{rr}1 / 2 & -1 / 3 \\ -3 / 8 & 3 / 4\end{array}\right]$, i.e., $T^{*} \delta_{1}=\frac{1}{2} \delta_{1}-\frac{1}{3} \delta_{2}$ and $T^{*} \delta_{2}=\frac{-3}{8} \delta_{1}+\frac{3}{4} \delta_{2}$ (where $\delta_{1}=$ Dirac mass at 1 , etc.). Then

$$
F(T)=\left\{x\left[\begin{array}{c}
1 \\
-3 / 2
\end{array}\right]: x \text { scalar }\right\}, \quad F\left(T^{*}\right)=\left\{x\left(\frac{3}{7}, \frac{-4}{7}\right): x \text { scalar }\right\},
$$

and an extreme in $F_{1}\left(T^{*}\right)$ is $m=\left(\frac{3}{7}, \frac{-4}{7}\right)$. Then $\varphi_{m}=\left[\begin{array}{c}1 \\ -1\end{array}\right]$, which bears no relation to the invariant function $f(1)=1, f(2)=\frac{-3}{2}$.

2.5. Lemma. Assume there exists $\alpha$ in $F(T)$ such that $\alpha \neq 0$ on $M$. If $m$ is in ext $F_{1}\left(T^{*}\right)$, then $x$ in $\operatorname{supp}(|m|)$ implies $\operatorname{supp}\left(T^{*} \delta_{x}\right) \subset \operatorname{supp}(|m|)$. (We shall write $t_{x}=T^{*} \delta_{x}$.)

Proof. If not, let $y$ be in $\operatorname{supp}\left(\left|t_{x}\right|\right) \backslash \operatorname{supp}(|m|)$ and choose $f$ in $C(X)$ with $0 \leqslant f \leqslant 1, f=1$ on $\operatorname{supp}(|m|)$, and $f(y)<1$. Let $g_{m}$ be a continuous extension of $\bar{\varphi}_{m} \mid \operatorname{supp}(|m|)$ to all $X$ such that $\left|g_{m}\right| \leqslant 1$. Since $\left|f(y) g_{m}(y)\right|<1$, we have 
$\left|T\left(f g_{m}\right)(x)\right|=\left|\int f g_{m} d t_{x}\right|<1$. Since $x$ is in $\operatorname{supp}(|m|)$, we have

$$
\left|\int T\left(f g_{m}\right) d m\right|<|m|(X)=1
$$

while $\int f g_{m} d m=\int \bar{\varphi}_{m} d m=\int d|m|=|m|(X)=1$. But this contradicts $T^{*} m=m$.

2.6. Lemma. Under the hypothesis of 2.5 , if $f \mid M=0$, then $T f \mid M=0$, so that the $T_{0}$ of the Introduction is well defined.

Proof. First let $m$ be in ext $F_{1}\left(T^{*}\right)$, so $f=0$ on $\operatorname{supp}(|m|)$. It $x$ is in $\operatorname{supp}(|m|)$, then by $2.5 \operatorname{supp}\left(t_{x}\right) \subset \operatorname{supp}(|m|)$, so $f=0$ on $\operatorname{supp}\left(t_{x}\right)$, whence $T f(X)=0$. Thus $T f=0$ on $\operatorname{supp}(|m|)$. But Krein-Milman easily implies that

$$
M=\text { closure } \bigcup\left\{\operatorname{supp}(|m|): m \text { in ext } F_{1}\left(T^{*}\right)\right\},
$$

so $T f=0$ on $M$.

2.7. THE FUNCTION $\psi$. For the rest of the section we assume the existence of a continuous $\psi$ which never vanishes, and satisfies $T \psi^{-1}=\psi^{-1}$. By 2.2 if $m$ is extreme in $F_{1}\left(T^{*}\right)$, then on $\operatorname{supp}(|m|)$ we have $\psi^{-1}=\int \psi^{-1} d m \bar{\varphi}_{m}$, and $\psi=a_{m} \varphi_{m}$, where $a_{m}=\left(\int \psi^{-1} d m\right)^{-1}$.

(Such a function $\psi$ need not exist. For example, let $X=R \cup\{\infty\}$ (the one-point compactification of the reals), and let $T f(x)=\frac{1}{2}(f(x)-f(-x)), T f(\infty)=0$. Then $T^{2}=T, M=X$ (since each measure of the form $\frac{1}{2}\left(\delta_{x}-\delta_{-x}\right)$ is invariant), and $T f=f$ implies $f(0)=f(\infty)=0$.)

2.8. The OPERATOR $R$. Let $R f=T\left(f \psi^{-1}\right) \psi$. It is easy to check that $R$ satisfies condition (1) of the introduction and $R^{n} f=T^{n}\left(f \psi^{-1}\right) \psi, R_{n} f=T_{n}\left(f \psi^{-1}\right) \psi$. Thus $R$ is strongly ergodic iff $T$ is strongly ergodic. Further properties of $R$ are listed in

2.9. Proposition. (a) $F(R)=\psi F(T)$.

(b) Let $W: F\left(T^{*}\right) \rightarrow C(X)^{*}$ be defined by $W(m)=\psi^{-1} d m$. Then $W$ is a bijection from $F\left(T^{*}\right)$ to $F\left(R^{*}\right)$, and if $m$ is in ext $F_{1}\left(T^{*}\right)$, then $W(m)=a_{m}^{-1} d|m|\left(a_{m}\right.$ as in 2.7).

(c) $R$ induces an operator $R_{0}$ on $C(M)$ which is Markov. Further, $f$ in $C(M)$ satisfies $R_{0} f=f$ iff $f$ is constant on $\operatorname{supp}(|m|)$ whenever $m$ is extreme in $F_{1}\left(T^{*}\right)$.

Proof. (a) is an exercise.

(b) To show that $W$ maps $F\left(T^{*}\right)$ into $F\left(R^{*}\right)$, let $m$ be in $F\left(T^{*}\right)$ and $f$ in $C(X)$. Then

$$
\int R f d W(m)=\int T\left(f \psi^{-1}\right) \psi \psi^{-1} d m=\int T\left(f \psi^{-1}\right) d m=\int f \psi^{-1} d m=\int f d W(m),
$$

so $W(m)$ is in $F\left(R^{*}\right)$. $W$ is a bijection, since the same argument shows that $W^{-1}(m)=\psi d m$ maps $F\left(R^{*}\right)$ into $F\left(T^{*}\right)$.

If $m$ is extreme, then on $\sup |m|, \psi^{-1}=a_{m}^{-1} \bar{\varphi}_{m}$, so for $f$ in $C(X)$,

$$
\int f d W(m)=\int f \psi^{-1} d m=a_{m}^{-1} \int f \bar{\varphi}_{m} d m=a_{m}^{-1} \int f d|m|,
$$

so $W(m)=a_{m}^{-1} d|m|$. 
(c) $R_{0}$ is well defined, because if $f \mid M=0$, then $f \psi^{-1} \mid M=0$, so $R f \mid M=$ $T\left(f \psi^{-1}\right) \psi \mid M=0$ by 2.6. To show $R_{0}$ is Markov, first, since $T \psi^{-1}=\psi^{-1}$ on $M$,

$$
R_{0} 1(x)=T_{0}\left(\psi^{-1}\right)(x) \psi(x)=\psi^{-1}(x) \psi(x)=1,
$$

so $R_{0} 1=1$. Second, if $x$ is in $\operatorname{supp}(|m|)$, where $m$ is extreme, and $f$ is in $C(M)$, then since $\operatorname{supp}\left(t_{x}\right) \subset \operatorname{supp}(|m|)$, we have

$$
\begin{aligned}
\left|R_{0} f(x)\right| & =\left|\int f \psi^{-1} d t_{x} \psi(x)\right|=\left|\int f a_{m}^{-1} \bar{\varphi}_{m} d t_{x} a_{m} \varphi_{m}(x)\right| \\
& =\left|\int f \bar{\varphi}_{m} d t_{x}\right| \leqslant\|f\|\left\|\varphi_{m}\right\|\left\|t_{x}\right\| \leqslant\|f\| .
\end{aligned}
$$

Thus if $x$ is in $\operatorname{supp}(|m|)$, then $\left\|R_{0}^{*} \delta_{x}\right\| \leqslant 1$, and since $R_{0}^{*} \delta_{x}(X)=R_{0} 1(x)$, it is a probability measure. By the density of such $x$ in $M, R_{0}$ is Markov.

Finally to describe $F\left(R_{0}\right)$, it is clear that by (a), $F\left(R_{0}\right)=\psi_{0} F\left(T_{0}\right)$, where $\psi_{0}=\psi \mid M$, so if $f$ is in $F\left(R_{0}\right)$, it has the form $f=\psi_{0} g$ with $g$ in $F\left(T_{0}\right)$. If $m$ is extreme, $g=k \bar{\varphi}_{m}$ on $\sup (|m|)$ by 2.2 , and, hence, $f=\psi g=a_{m} \varphi_{m} k \bar{\varphi}_{m}=a_{m} k$, a constant on $\sup (|m|)$. Conversely, if $f \in C(M)$ and is constant on each sup $|m|$, say $f=k_{m}$, then since, by $2.5, x$ in sup $|m| \operatorname{implies} \sup \left(t_{\mathrm{x}}\right) \subset \sup (|m|)$, we have for $x$ in $\sup (|m|)$ that

$$
\begin{aligned}
R f(x) & =T\left(f \psi^{-1}\right)(x) \psi(x)=\int f \psi^{-1} d t_{x} \psi(x)=k_{m} \int \bar{\varphi}_{m} d t_{x} \varphi_{m}(x) \\
& =k_{m} \bar{\varphi}_{m}(x) \varphi_{m}(x)=k_{m}
\end{aligned}
$$

(since $T \bar{\varphi}_{m}=\bar{\varphi}_{m}$ on $\left.\sup (|m|)\right)$. Thus $R_{0} f=f$ on $\sup (|m|)$ and, by the usual Krein-Milman argument, we have $R_{0} f=f$ on $M$. (Note that when $\psi$ exists, $R_{0}$ is uniquely determined by $T$, although $R$ need not be, since if $x$ is $\operatorname{supp}(|m|)$, we have $R_{0} f(x)=T\left(f \bar{\varphi}_{m}\right)(x) \varphi_{m}(x)$.)

2.10. WorKeD EXAMPLE. Let $X=\{1,2,3\}$ and let $T$ be given by

$$
\left[\begin{array}{ccc}
1 / 2 & -1 / 2 & 0 \\
-1 / 4 & 3 / 4 & 0 \\
0 & 0 & 1
\end{array}\right]
$$

Then

$$
F(T)=\left\{x\left[\begin{array}{c}
1 \\
-1 \\
0
\end{array}\right]+y\left[\begin{array}{l}
0 \\
0 \\
1
\end{array}\right]: x, y \text { scalar }\right\}
$$

and

$$
F\left(T^{*}\right)=\left\{x\left(\frac{1}{3}, \frac{-2}{3}, 0\right)+y(0,0,1): x, y \text { scalar }\right\} .
$$

Extreme measures are $m_{1}=\left(\frac{1}{3}, \frac{-2}{3}, 0\right)$ and $m_{2}=(0,0,1)$. Let

$$
\psi^{-1}=\left[\begin{array}{c}
a \\
-a \\
b
\end{array}\right], \quad a \text { and } b \text { nonzero. }
$$


Then $R$ is the matrix gotten by eliminating the minus signs in the $T$ matrix (and hence is independent of $a$ and $b), F(R)=\psi F(T)$ is gotten by eliminating minus signs in the formula for $F(T)$, and likewise for $F\left(R^{*}\right)$.

2.11. ReMARK. Let $B_{0}=W\left(F_{1}\left(T^{*}\right)\right)$, a weak-* compact convex subset of $F\left(R^{*}\right)$. Since $W$ is a convex function and a bijection on $F_{1}\left(T^{*}\right), m$ is extreme in $F_{1}\left(T^{*}\right)$ iff $W(m)=a_{m}^{-1} d m$ is extreme in $B_{0}$. By Krein-Milman $B_{0}$ is the weak-* closed convex hull of these, i.e., measures of the form $k p$, where $p$ is a probability. This leads to the hope that ergodic properties of Markov operators based on the analysis of invariant probabilities (see, e.g., $\left[\mathbf{A}, \mathbf{S}_{\mathbf{1}}, \mathbf{S}_{\mathbf{3}}\right]$ ) will in some sense be shared by the operator $T$.

3. Ergodic theorems. For the Markov case, Theorem 3.2 was proved by Robert Sine $\left[\mathbf{S}_{\mathbf{3}}\right.$, Theorem 3]. The argument given there is apparently incomplete, although Professor Sine has pointed out to me that the argument goes through after some corrections (e.g., change "Baire" to "Borel"). The proof we give here is new.

3.1. Definition. Let $\mathbf{E}=\left\{|m|: m \in \operatorname{ext}\left(F_{1}\left(T^{*}\right)\right)\right\}$. $T$ is continuously scattered if there is a family of continuous functions, each constant on the support of each element of $\mathbf{E}$, and sufficient to separate these sets. (If $T$ is Markov, then each member of the family will be $T$-invariant on $M$, but this does not hold in general.)

3.2. TheOREM (CF. [ $\mathbf{S}_{3}$, THEOREM 3]). Let $T$ be a contraction on $C(X)$ and assume there exists $\alpha$ in $C(X)$ such that $T \alpha=\alpha$ and $\alpha \neq 0$ on $M$. Then $T$ is locally strongly ergodic iff it is continuously scattered.

Proof. Assume $T$ is locally s.e., i.e., $T_{0}$ is strongly ergodic on $C(M)$. If $\psi^{-1}=\alpha \mid M$, then $T_{0}$ satisfies condition 2.7 and, by $2.9(\mathrm{c})$, the induced operator $R_{0}$ is Markov and, by 2.8 , strongly ergodic. By $2.9(\mathrm{~b})$, elements of $\mathbf{E}$ are extreme invariant probabilities for $R_{0}$, and the existence of the separating family follows from $\left[\mathbf{S}_{\mathbf{3}}\right.$, Theorem 3].

Conversely, suppose $T$ is continuously scattered. Let $\mathbf{F}$ be the closed selfadjoint subalgebra of $C(M)$ which is generated by restrictions to $M$ of the separating family plus $1_{M}$. By $2.9(\mathrm{c}), \mathrm{F} \subset F\left(R_{0}\right)$. To prove $R_{0}$ is strongly ergodic it suffices, by the separation criterion of the Introduction, to show that $\mathbf{F}$ separates $F\left(R_{0}^{*}\right)$ or, equivalently, $F\left(R_{0}^{*}\right) \cap \mathbf{F}^{\perp}=(0)$.

We show that $\mathbf{F}$ is a lattice. Let $\mathbf{D}$ be the equivalence class decomposition of $M$ determined by the algebra $\mathbf{F}$, and let $Y$ be the quotient space $M / \mathbf{D}$, furnished with the quotient topology, which is compact $T_{2}$ (cf. $\left[\mathbf{S}_{3}\right]$ ). This topology contains the weak topology on $M / \mathbf{D}$ determined by functions in $\mathbf{F}$ and, since the latter topology is $T_{2}$, the two topologies are equal. By Stone-Weierstrass, $\mathbf{F}$ is essentially $C(M / D)$, and so $\mathbf{F}$ is a lattice.

As a first step to proving that $F\left(R_{0}^{*}\right) \cap \mathbf{F}^{\perp}=(0)$, we show that if $m$ is an extreme point of the unit ball of $F\left(R_{0}^{*}\right) \cap \mathbf{F}^{\perp}$, then $\operatorname{supp}(|m|)$ is contained in some $D \in \mathbf{D}$. Suppose not. Because $\mathbf{F}$ is a selfadjoint subalgebra, there exist $f$ in $\mathbf{F}$ and $c$ in $\mathbf{R}$ such that if $A=\{x: f(x) \geqslant c\}$ and $B=M \backslash A$, then $|m|(A)>0$ and $|m|(B)>0 . A$ and $B$ are Baire sets in $M$ and, in fact, there exist bounded sequences $f_{n} \in \mathbf{F}$, 
$g_{n} \in \mathbf{F}$ with $f_{n}(x) \rightarrow 1_{A}(x), g_{n}(x) \rightarrow 1_{B}(x)$ for all $x$ in $M$. (E.g., let $g(x)=$ $(f(x)+\|f\|) / 2\|f\|$ and $d=(c+\|f\|) / 2\|f\|$. Then $g$ is in $\mathbf{F}, 0 \leqslant g \leqslant 1$, and $d \neq 0$, since $A \neq M$. Now $A=\{x: g(x) \geqslant d\}$ and, since $\mathbf{F}$ is a lattice, we can let $f_{n}(x)=(\min (g(x), d) / d)^{n}, g_{n}(x)=1-f_{n}(x)$.

If $k$ is a bounded Baire function on $M$, one defines $R_{0} k(x)=\int k d r_{x}$. From a standard result on transition probabilities, $R_{0} k$ is a Baire function. Since $R_{0}^{*} m=m$, and easy transfinite induction shows that for $k$ Baire, $\int R_{0} k d m=\int k d m$. So since $1_{A}$ is Baire, $R_{0} 1_{A}$ is defined and, in fact, $R_{0} 1_{A}(x)=1_{A}(x)$ for $x$ in $M$, because

$$
R_{0} 1_{A}(x)=\int 1_{A} d r_{x}=\lim \int f_{n} d r_{x}=\lim R_{0} f_{n}(x)=\lim f_{n}(x)=1_{A}(x),
$$

the next to the last step because $f_{n} \in \mathbf{F} \subset F\left(R_{0}\right)$. Hence $1_{A}(x)=r_{x}(A)$, i.e., $r_{x}$ is supported by $A$ if $x$ is in $A$, and by $B$ if $x$ is in $B$. This implies that for all $g$ in $C(M)$,

$$
R_{0}\left(1_{A} g\right)(x)=\int_{A} g d r_{x}=1_{A}(x) R_{0} g(x) .
$$

Let $t=|m|(A)$ and define $m_{A}(Z)=m(Z \cap A) / t, m_{B}(Z)=m(Z \cap B) /(1-t)$, so $m=t m_{A}+(1-t) m_{B}$. Then $m_{A} \neq m_{B}$, and we achieve a contradiction to extremeness if we show that $m_{A}$ and $m_{B}$ are in the unit ball of $F\left(R_{0}^{*}\right) \cap \mathbf{F}^{\perp}$. To show $m_{A}$ is in $F\left(R_{0}^{*}\right)$ (and likewise for $\left.m_{B}\right)$, if $g$ is in $C(M)$, then

$$
\int R_{0} g d m_{A}=\frac{1}{t} \int 1_{A} R_{0} g d m=\frac{1}{t} \int R_{0}\left(1_{A} g\right) d m=\frac{1}{t} \int 1_{A} g d m=\int g d m_{A} .
$$

(In the next to the last step we used $\int R_{0} k d m=\int k d m$ for $k$ Baire.)

To show $m_{A}$ is in $\mathbf{F}^{\perp}$, let $g$ be in $\mathbf{F}$. Since $\mathbf{F}$ is an algebra and $1_{A}(x)=\lim f_{n}(x)$ with $f_{n}$ in $\mathbf{F}$, we have $\int g d m_{A}=\lim (1 / t) \int g f_{n} d m=0$, since $m$ is in $\mathbf{F}^{\perp}$.

The contradiction shows that $\operatorname{supp}(|m|) \subset D$ for some $D$ in D. By the continuously scattered hypothesis, $D$ supports only one measure of the form $|r|$, where $r$ is in ext $F_{1}\left(T^{*}\right)$. In fact this implies that the space of elements of $F\left(T^{*}\right)$ supported by $D$ is one dimensional. To show this, suppose $s$ is in ext $F_{1}\left(T^{*}\right)$ and supported by $D$. Then $|s|=|r|$, or $\bar{\varphi}_{s} s=\bar{\varphi}_{r} r$. Since $\operatorname{supp}(|r|)=\operatorname{supp}(|s|), 2.2$ and 2.3 imply that for some scalar $k, \bar{\varphi}_{s}=k \bar{\varphi}_{r}$ on supp $(|r|)$, whence $s=k r$. By Krein-Milman, if $F\left(T^{*}\right)_{D}$ $=$ elements of $F\left(T^{*}\right)$ supported by $D$, then all elements in the unit ball of $F\left(T^{*}\right)_{D}$ are multiples of $r$, and so $F\left(T^{*}\right)_{D}$ is one dimensional. (Implicit in this is the easily proved fact that $r$ is extreme in $F_{1}\left(T^{*}\right)_{D}$ iff it is an extreme point of $F_{1}\left(T^{*}\right)$ with support in $D$.) Now if $F\left(R^{*}\right)_{D}$ denotes the elements of $F\left(R^{*}\right)$ supported by $D$, then $F\left(R^{*}\right)_{D}=W\left(F\left(T^{*}\right)_{D}\right)$, and so $F\left(R^{*}\right)_{D}$ is one dimensional. So the measure $m$ must have the form $m=W(k r)=k a_{r}^{-1}|r|$, where $r$ is extreme in $F_{1}\left(T^{*}\right)$. Since $m$ is also in $\mathbf{F}^{\perp}$ and each element of $\mathbf{F}$ is constant on $D$, we get $k=0$, and so $m=0$. Thus $F\left(R_{0}^{*}\right) \cap \mathbf{F}^{\perp}=(0)$.

From now on we assume the existence of $\alpha$ as in the hypotheses of 3.2.

3.3. TheOREM (CF. [ $\mathbf{S}_{3}$, THEOREM 4]). Suppose there exists on $C(M)$ a strongly ergodic contraction $S$ such that $F\left(T_{0}^{*}\right)=F\left(S^{*}\right)$. Then $T$ is locally s.e. 
Proof. In view of 2.6, we may assume $X=M$, by looking at $T_{0}$. If $m$ is extreme in $F_{1}\left(T^{*}\right), m=\varphi_{m}|m|$, then $\psi=a_{m} \varphi_{m}$ on $\operatorname{supp}(|m|)$, and since $F_{1}\left(T^{*}\right)=F_{1}\left(S^{*}\right)$, we have by 2.2 that $S \psi^{-1}=\psi^{-1}$ on $\operatorname{supp}(|m|)$, hence on $M=X$. Since $S$ is s.e. and satisfies the hypothesis of 3.2 , it is continuously scattered, and hence so is $T$. By 3.2 again, $T$ is locally s.e.

3.4. TheOREM (CF. [A, THEOREM 3.1]). Suppose there exists a contractive projection $S$ such that $T_{n} f \rightarrow 0$ iff $S f=0$. Then $T$ is locally s.e.

Proof. As in Remark 2.1 and Lemma 2.2 of $[\mathbf{A}]$, we prove $F_{1}\left(T^{*}\right)=F_{1}\left(R^{*}\right)$, and then apply 3.3 .

3.5. Theorem (CF. [ $\mathbf{S}_{1}$, TheOREMS 2.2, 2.7]). Assume (a) $\psi^{-1} \in F(T)$ and (b) $|\psi(x)|=1$ for all $x$. Then $T$ is strongly ergodic iff $\psi F(T)$ separates the probabilities in $\psi^{-1} F\left(T^{*}\right)=W\left(F\left(T^{*}\right)\right)$.

Proof. $R$ is a Markov operator, since by (a), $R 1=T\left(\psi^{-1}\right) \psi=\psi^{-1} \psi=1$, and by (b), $\|R\| \leqslant\|\psi\|\left\|\psi^{-1}\right\|=1$. Since $F(R)=\psi F(T)$ and $F\left(R^{*}\right)=\psi^{-1} F\left(T^{*}\right)$, our result is just [ $\mathbf{S}_{1}$, Theorems 2.2 and 2.7] applied to $R$.

3.6. REMARK. If (a) and (b) of 3.5 hold, then since $F(T)$ is the range of a contractive projection, [F-R, Theorem 4] implies that $F(T)$ is isometrically *-isomorphic to $C(K)$ for some compact $K$.

ACKNOWLEDGEMENTS. I am indebted to the authors of [F-R] for sending me a preprint, which was the inspiration for the present paper.

I am also indebted to the referee for the detection of a number of errors.

ADDED IN PROOF. I can now prove Lemma 2.5 without the assumption that there exists $\alpha$ in $F(T)$ with $\alpha \neq 0$ on $M$. This leads to a weakening of hypotheses elsewhere in the paper, e.g., in Theorem 3.2 we need only assume that $T \alpha=\alpha$ on the set $M$ rather than on all of $X$.

\section{REFERENCES}

[A] R. Atalla, On the mean convergence of Markov operators, Proc. Edinburgh Math. Soc. (2) 19 (1974), 205-209.

[F-R] Y. Friedman and B. Russo, Contractive projections on $C_{0}(K)$. Trans. Amer. Math. Soc. 273 (1982), 57-73.

[LI] S. Lloyd, On the mean ergodic theorem of Sine, Proc. Amer. Math. Soc. 56 (1976), 121-126.

[Sa] R. Sato, The Hahn-Banach theorem implies Sine's mean ergodic theorem, Proc. Amer. Math. Soc. 77 (1979), 426.

$\left[\mathbf{S}_{\mathbf{1}}\right]$ R. Sine, Geometric theory of a single Markov operator, Pacific J. Math. 27 (1968), 155-166.

$\left[\mathbf{S}_{2}\right]$, A mean ergodic theorem, Proc. Amer. Math. Soc. 24 (1970), 438-439.

$\left[\mathbf{S}_{3}\right]$ - On local uniform mean convergence for Markov operators, Pacific J. Math. 60 (1975), $247-252$.

Department of Mathematics, Ohio University, Athens, Ohio 45701 